\title{
Epidemiological, genetic, and clinical characterization by age of newly diagnosed acute myeloid leukemia based on an academic population-based registry study (AMLSG BiO)
}

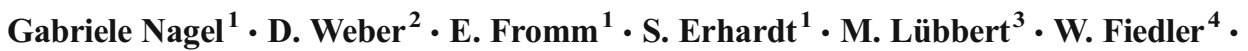 \\ T. Kindler ${ }^{5}$ - J. Krauter ${ }^{6}$ P. Brossart ${ }^{7}$ - A. Kündgen ${ }^{8}$ • H. R. Salih ${ }^{9} \cdot$ J. Westermann ${ }^{10}$. \\ G. Wulf ${ }^{11}$ - B. Hertenstein ${ }^{12}$ - M. Wattad ${ }^{13}$ - K. Götze ${ }^{14}$ - D. Kraemer ${ }^{15}$. T. Heinicke ${ }^{16}$. \\ M. Girschikofsky ${ }^{17}$ - H.G. Derigs ${ }^{18}$ - H. A. Horst ${ }^{19}$ - C. Rudolph ${ }^{2}$ - M. Heuser ${ }^{20}$ • \\ G. Göhring ${ }^{21} \cdot$ V. Teleanu ${ }^{2}$ - L. Bullinger ${ }^{2}$ - F. Thol ${ }^{20}$ • V. I. Gaidzik ${ }^{2} \cdot$ P. Paschka $^{2}$. \\ K. Döhner ${ }^{2}$ • A. Ganser ${ }^{20}$ • Hartmut Döhner ${ }^{2}$ - R. F. Schlenk ${ }^{2,22} \cdot$ German-Austrian $^{2}$ \\ AML Study Group (AMLSG)
}

Received: 17 August 2017 / Accepted: 16 September 2017 / Published online: 31 October 2017

(C) The Author(s) 2017. This article is an open access publication

Abstract We describe genetic and clinical characteristics of
acute myeloid leukemia (AML) patients according to age

Electronic supplementary material The online version of this article (https://doi.org/10.1007/s00277-017-3150-3) contains supplementary material, which is available to authorized users.

Gabriele Nagel

gabriele.nagel@uni-ulm.de

Hartmut Döhner

hartmut.doehner@uniklinik-ulm.de

1 Institute of Epidemiology and Medical Biometry, Ulm University, Helmholtzstr. 22, 89081 Ulm, Germany

2 Department of Internal Medicine III, University Hospital Ulm, Albert-Einstein-Allee 23, 89081 Ulm, Germany

3 Department of Internal Medicine I, Faculty of Medicine, University Hospital Freiburg, Freiburg, Germany

4 Department of Internal Medicine II, University Hospital Hamburg-Eppendorf, Hamburg, Germany

5 Department of Internal Medicine III, University Medical Center Mainz, Mainz, Germany

6 Department of Internal Medicine III, Hospital Braunschweig, Braunschweig, Germany

7 Department of Internal Medicine III, University Hospital of Bonn, Bonn, Germany

8 Department of Hematology, Oncology and Clinical Immunology, University Hospital of Düsseldorf, Düsseldorf, Germany

9 Department of Internal Medicine II, University Hospital of Tübingen, Tübingen, Germany

10 Department of Hematology, Oncology and Tumor Immunology, Charité - Campus Virchow Clinic, Berlin, Germany from an academic population-based registry. Adult patients with newly diagnosed AML at 63 centers in Germany and
11 Department of Hematology and Oncology, University Hospital of Göttingen, Göttingen, Germany

12 Department of Internal Medicine I, Hospital Bremen-Mitte, Bremen, Germany

13 Department of Hematology and Oncology, Hospital Essen-Werden, Essen, Germany

14 Department of Internal Medicine III, University Hospital Klinikum rechts der Isar, Munich, Germany

15 Department of Oncology and Hematology, Hospital Oldenburg, Oldenburg, Germany

16 Department of Hematology and Oncology, University Hospital of Magdeburg, Magdeburg, Germany

17 Department of Hematology and Oncology, Hospital Elisabethinen Linz, Linz, Austria

18 Department of Internal Medicine III, Hospital Frankfurt-Hoechst, Frankfurt, Germany

19 Department of Internal Medicine II, University Hospital of Schleswig-Holstein, Kiel, Germany

20 Department of Hematology, Hemostasis, Oncology, and Stem Cell Transplantation, Hannover Medical School, Hannover, Germany

21 Institute of Human Genetics, Hannover Medical School, Hannover, Germany

22 NCT Trial Center, National Center for Tumor Diseases, Heidelberg, Germany 
Austria were followed within the AMLSG BiO registry (NCT01252485). Between January 1, 2012, and December 31,2014 , data of 3525 patients with AML ( $45 \%$ women) were collected. The median age was 65 years (range 18-94). The comparison of age-specific AML incidence rates with epidemiological cancer registries revealed excellent coverage in patients $<70$ years old and good coverage up to the age of 80. The distribution according to the European LeukemiaNet (ELN) risk categorization from 2010 was $20 \%$ favorable, $31 \%$ intermediate- $1,28 \%$ intermediate- 2 , and $21 \%$ adverse. With increasing age, the relative but not the absolute prevalence of patients with ELN favorable and intermediate-1 risk $(p<0.001)$, with activating FLT3 mutations $(p<0.001)$, with ECOG performance status $<2(p<0.001)$, and with HCT-CI comorbidity index $<3(p<0.001)$ decreased. Regarding treatment, obesity and favorable risk were associated with an intensive treatment, whereas adverse risk, higher age, and comorbidity index $>0$ were associated with non-intensive treatment or best supportive care. The AMLSG BiO registry provides reliable population-based distributions of genetic, clinical, and treatment characteristics according to age.

Keywords Epidemiology · Genetics · Older age · AML · Registry

\section{Introduction}

Acute myeloid leukemia (AML) is the most frequent acute leukemia in adults with an incidence of 3 to 4 per 100,000 persons per year $[1,2]$. The median age at diagnosis ranges from 66 to 71 years $[1,3,4]$. Of note, over the last years the incidence has remained stable in younger patients but significantly increased in patients aged over 75 years $[3,5]$. AML is a genetically very heterogeneous disorder characterized by the accumulation of somatically acquired genetic changes in hematopoietic progenitor cells that alter normal mechanisms of self-renewal, proliferation, and differentiation [6]. Treatment approaches are influenced by various factors, including patient features such as age, comorbidities, body mass index (BMI), and performance status as well as disease characteristics whereby the genetic profile of the disease is the most important prognostic factor [3, 7-11].

Scientific and technical advances accelerate the development and application of molecular genetic testing in subjects with leukemia. Mutations in the genes such as nucleophosmin1 (NPM1), FMS-related tyrosine kinase 3 (FLT3), and CCAAT/ enhancer-binding protein alpha $(C E B P A)$ in cytogenetically normal AML influence the prognosis of AML patients [12] and have entered clinical routine [11, 13]. Activating FLT3 mutations including internal tandem duplication (ITD) as well as tyrosine kinase domain (TKD) mutations and point mutations in exone 12 of NPM1 are reported as frequent mutations in AML in an average young trial population with reported incidences of 33 and $28 \%$, respectively [8], whereas mutations in CEBPA are less frequent [12]. In 2010, the European LeukemiaNet (ELN) proposed a standardized reporting system integrating cytogenetic and molecular genetic data which recently has been updated [13]. In addition, the efficacy and availability of FLT3 inhibitors mark the starting point beyond acute promyelocytic leukemia of genotype-directed therapy in AML. Recently, the multi-kinase inhibitor midostaurin has shown efficacy in a randomized phase III trial of patients with activating FLT3 mutations [14].

The introduction of personalized medicine will pose new challenges with respect to transition into clinical routine and in the evaluation of new treatment approaches in small genetically defined subgroups $[8,15]$. Most of our knowledge on leukemia has been derived from center- and cooperative group-based clinical data rather than population-based registries $[4,16,17]$. Due to this increasing heterogeneity, registries are a valuable data source to appraise the presenting clinical characteristics and treatment decision in AML patients [4]. There is evidence from population-based registries that the survival expectations of patients with AML have improved over the past decades [17, 18], whereby in older AML patients only little or no progress has been made $[5,19,20]$. This difference probably reflects the difference in disease profile and frequency as well as severity of comorbidities in older compared to younger patients $[3,9,13]$. However, our understanding of genetic heterogeneity according to patients' demographic, clinical, and treatment characteristics is still incomplete $[17,18]$.

The objectives of our study were to investigate epidemiological, genetic, and clinical characteristics of patients participating in the German-Austrian AML Study Group registry study (AMLSG BiO; ClinicalTrials.gov Identifier: NCT01252485) from 2012 to 2014, to compare them with selected epidemiological cancer registry data and to analyze distributions of genetic, clinical, and treatment characteristics according to age.

\section{Material and methods}

\section{Study population}

The AMLSG BiO registry study was initiated in 2010, and activation of most sites was completed in 2011. We aimed to analyze epidemiological, genetic, and clinical features between 2012 and 2014, representing a period of 2 years with full recruitment. We intended to register all patients aged 18 years or older with newly diagnosed AML at all centers of the German-Austrian AML Study Group (AMLSG) within the AMLSG BiO registry study. Via a web-based system, 
participating AMLSG centers registered patients with newly diagnosed AML based on local bone marrow and peripheral blood assessment after written informed consent. In all patients, bone marrow and peripheral blood samples were sent overnight by courier service to the AMLSG reference laboratories for cytogenetic and molecular genetic analyses (University of Ulm, Hannover Medical School). The study was approved by the ethical review boards of all participating centers.

For the current analysis, 3521 AML cases diagnosed between January 1, 2012, and December 31, 2014, were identified in the AMLSG BiO registry study database. Data were collected on sex, age, date of diagnosis, Eastern Cooperative Oncology Group (ECOG) performance status [21], and comorbidities according to the Hematopoietic Cell TransplantationSpecific Comorbidity Index (HCT-CI) [22]. AML cases were classified according to the 2008 World Health Organization (WHO) proposal [23] and risk-stratified according to the 2010 ELN classification [11]. In 3213 (91\%) patients, information on treatment strategy was available including intensive chemotherapy, non-intensive treatment (azacitidine, decitabine, low-dose cytarabine), and best supportive care (BSC).

\section{Cytogenetics and molecular genetics}

Chromosome banding analysis (within 14 days) and molecular screening (within $48 \mathrm{~h}$ ) were performed in the two AMLSG central laboratories in Hannover and Ulm. Karyotypes were described according to the International System for Human Cytogenetic Nomenclature [24]. Leukemia samples were analyzed for mutations in FLT3 (ITDs, and tyrosine kinase domain [TKD] mutations at codons D835/I836), NPM1, and CEBPA (both monoallelic and biallelic) as previously described $[12,25,26]$.

The molecular profile of the disease in conjunction with a recommendation of potential trial participation was documented in a web-based system, and results were communicated immediately via facsimile to the participating centers.

\section{Statistical methods}

Age-standardized incidence rates (ASR) of AML for Germany $(N=3251)$ were calculated using German population data in the years 2012-2014 [27]. Based on the geographic distribution of the contributing centers (Supplemental Fig. 1) with no or only few centers in MecklenburgPommerania, Brandenburg, Thuringia, Saxony, and Bavaria, the data from these regions were excluded from the denominator of the population data.

For the AML diagnosis (ICD-9: 205.0 or ICD-10 C92.0), the incidence rates by sex and 5-year age classes were provided by the following cancer registries: Saarland (2006-2010), Bavaria (2010-2012), and North Rhine-Westphalia (2010-
2013). Based on the data, mean annual incidence rates were calculated. In addition, data on AML from the Surveillance, Epidemiology, and End Results (SEER) program in the USA was used [28].

Multinomial logistic regression was calculated to estimate odds ratios (OR) and the $95 \%$ confidence intervals $(95 \% \mathrm{CI})$ for treatment strategy (reference: intensive chemotherapy). The covariates age class, sex, ECOG performance status, ELN risk category, HCT comorbidity index, and BMI $(<20$, $20-24,>25 \mathrm{~kg} / \mathrm{m}^{2}$ ) were entered simultaneously as independent variables into the final model to determine their effects on treatment decision.

\section{Results}

Between 2012 and 2014, 3521 patients with newly diagnosed AML (45\% women) were registered. The baseline characteristics of the AMLSG BiO study population are described in Table 1. Overall, the median age was 65 years (Q1, Q3; 54, 74, range 18-94); men were slightly older than women (median age 66 vs. 64 years). Independent of gender, most AML cases were observed in the age group 70 years and older $(N=1396$, $39.7 \%)$. The age-specific incidence rates for AML in Germany are shown in Fig. 1a (for men), b (for women). The comparison of the age-specific AML incidence rates with data from German epidemiological cancer registries in Bavaria, North Rhine-Westphalia, and Saarland as well as the US SEER cancer database revealed good coverage of AML patients in the AMLSG BiO registry in younger and middle age classes, while older patients were less frequently registered. In both men and women, the incidence rates increased with age.

In the AMLSG $\mathrm{BiO}$ registry, most registered cases were defined as de novo AML (82.1\%), followed by secondary AML evolving after a myelodysplastic syndrome (13.6\%) and therapy-related AML (4.3\%). Mutated NPM1 was present in 914 patients (26\%), followed by FLT3-ITD in $666(18.9 \%)$ and FLT3-TKD mutations in 219 (6.2\%) cases. In women, FLT3-ITD and mutated NPM1 were more prevalent than in men (both $p$ values $<0.0001$ ). According to the 2010 ELN classification, patients were diagnosed with favorable $(N=534,20.2 \%)$, intermediate risk-1 $(N=819,31 \%)$, intermediate risk-2 $(N=735,27.8 \%)$, and adverse risk $(N=558$, $21.1 \%$ ). Categorization of cases according to the $2008 \mathrm{WHO}$ classification revealed AML with recurrent genetic abnormalities as the largest subgroup (41.2\%) including the two provisional entities AML with mutated NPM1 (25.3\%) and AML with mutated CEBPA (4.2\%), followed by AML not otherwise specified (30.5\%), AML with MDS-related changes $(24.5 \%)$, and therapy-related AML (3.8\%) (Fig. 2).

Absolute and relative prevalence according to age groups as well as according to the 2010 ELN risk groups, HCT 
Table 1 Study population AMLSG BiO registry 2012-2014

\begin{tabular}{|c|c|c|c|c|c|}
\hline & & $\begin{array}{l}\text { Total } \\
N=3,521 \\
\text { Median (Q1;Q3) }\end{array}$ & $\begin{array}{l}\text { Men } \\
N=1,939 \\
\text { Median (Q1;Q3) }\end{array}$ & $\begin{array}{l}\text { Women } \\
N=1,582 \\
\text { Median (Q1;Q3) }\end{array}$ & $p$-value* \\
\hline Age (years) & & $65(54 ; 74)$ & $66(55 ; 74)$ & $64(52 ; 74)$ & 0.0083 \\
\hline \multirow[t]{2}{*}{ BMI $\left(\mathrm{kg} / \mathrm{m}^{2}\right)$} & & $26(23 ; 29)$ & $26(24 ; 29)$ & $25(23 ; 29)$ & $<.0001$ \\
\hline & & $\mathrm{N}(\%)$ & $\mathrm{N}(\%)$ & $\mathrm{N}(\%)$ & \\
\hline \multirow[t]{4}{*}{ Age classes (years) } & $<45$ & 409 (11.62) & $212(51.83)$ & $197(48.17)$ & 0.0091 \\
\hline & $45-59$ & $840(23.86)$ & $428(50.95)$ & $412(49.05)$ & \\
\hline & $60-69$ & $876(24.88)$ & $501(57.19)$ & $375(42.81)$ & \\
\hline & $\geq 70$ & $1,396(39.65)$ & $798(57.16)$ & $598(42.84)$ & \\
\hline \multirow[t]{2}{*}{ Country } & Germany & $3,251(92.33)$ & $1,793(55.15)$ & $1,458(44.85)$ & 0.7322 \\
\hline & Austria & $270(7.67)$ & $146(54.07)$ & $124(45.93)$ & \\
\hline \multirow[t]{3}{*}{ Year of diagnosis } & 2012 & $994(28.23)$ & $556(55.94)$ & $438(44.06)$ & 0.7559 \\
\hline & 2013 & $1,209(34.34)$ & $657(54.34)$ & $552(45.66)$ & \\
\hline & 2014 & $1,318(37.43)$ & $726(55.08)$ & $592(44.92)$ & \\
\hline \multirow[t]{3}{*}{ Type } & de novo AML & $2891(81.1)$ & $1580(81.5)$ & $1311(82.9)$ & $<.0001$ \\
\hline & secondary AML & $478(13.6)$ & $295(15.2)$ & $183(11.6)$ & \\
\hline & therapy-related AML & $152(4.3)$ & $64(3.3)$ & $88(5.5)$ & \\
\hline Prevalence of FLT3-ITD & & $666(18.93)$ & $306(45.95)$ & $360(54.05)$ & $<.0001$ \\
\hline Prevalence of FLT3-TKD & & $219(6.23)$ & $109(49.77)$ & $110(50.23)$ & 0.1024 \\
\hline Prevalence of $N P M 1$ mutation & & $914(25.97)$ & $418(45.73)$ & $496(54.27)$ & $<.0001$ \\
\hline \multirow[t]{2}{*}{ Prevalence of $C E B P A$} & monoallelic & $86(5.40)$ & $40(46.51)$ & $46(53.49)$ & 0.0633 \\
\hline & biallelic mutation & $70(2.37)$ & $43(61.43)$ & $27(38.57)$ & \\
\hline \multirow[t]{4}{*}{2010 ELN classification } & Favorable & $534(20.18)$ & $275(51.50)$ & $259(48.50)$ & 0.4590 \\
\hline & Intermediate-1 & $819(30.95)$ & $452(55.19)$ & $367(44.81)$ & \\
\hline & Intermediate-2 & $735(27.78)$ & $409(55.65)$ & $326(44.35)$ & \\
\hline & Adverse & $558(21.09)$ & $308(55.20)$ & $250(44.80)$ & \\
\hline \multirow[t]{3}{*}{ HCT-Comorbidity Index } & 0 & $1,337(38.96)$ & $715(53.48)$ & $622(46.52)$ & 0.1364 \\
\hline & $1-2$ & $1,152(33.57)$ & $639(55.47)$ & $513(44.53)$ & \\
\hline & $\geq 3$ & $943(27.48)$ & $544(57.69)$ & $399(42.31)$ & \\
\hline \multirow[t]{5}{*}{ ECOG } & 0 & $1,416(40.38)$ & $777(54.87)$ & $639(45.13)$ & 0.7118 \\
\hline & 1 & $1,491(42.51)$ & $809(54.26)$ & $682(45.74)$ & \\
\hline & 2 & $452(12.89)$ & $262(57.96)$ & $190(42.04)$ & \\
\hline & 3 & $129(3.68)$ & $73(56.59)$ & $56(43.41)$ & \\
\hline & 4 & $19(0.54)$ & $11(57.89)$ & $8(42.11)$ & \\
\hline \multirow[t]{3}{*}{ Study participation } & Yes & $591(20.66)$ & $299(50.59)$ & $292(49.41)$ & 0.0255 \\
\hline & No & $2,270(79.34)$ & $1,265(55.73)$ & $1,005(44.27)$ & \\
\hline & & $\mathrm{N}=3,213$ & $\mathrm{~N}=1,760$ & $\mathrm{~N}=1,453$ & \\
\hline \multirow[t]{6}{*}{ Therapy } & Intensive & $2,268(71.12)$ & $1,223(53.92)$ & $1,045(46.08)$ & 0.5646 \\
\hline & $\mathrm{BSC}$ & $298(9.34)$ & $171(57.38)$ & $127(42.62)$ & \\
\hline & Non-intensive & & & & \\
\hline & AZA & $113(3.54)$ & $60(53.10)$ & $53(46.90)$ & \\
\hline & $\mathrm{DAC}$ & $243(7.62)$ & $142(58.44)$ & $101(41.56)$ & \\
\hline & LD AraC & $267(8.37)$ & $148(55.43)$ & $119(44.57)$ & \\
\hline
\end{tabular}

$A M L$ acute myeloid leukemia, $A Z A$ 5-azacytidine, $B M I$ body mass index, $B S C$ best supportive care, $C E B P A$ CCAAT/enhancer-binding protein alpha, $D A C$ decitabine, $E C O G$ Eastern Cooperative Oncology Group performance status, ELN European LeukemiaNet, FLT3 FMS-related tyrosine kinase 3 , $H C T$ hematopoietic cell transplantation, ITD internal tandem duplication, $L D$ AraC low-dose arabinoside cytosine, MDS myelodysplastic syndrome, $N$, number of patients, NPM1 nucleophosmin-1, $Q$ quartile, TKD tyrosine kinase domain

${ }^{\mathrm{a}} \mathrm{Chi}^{2}$ test for categorical and Wilcoxon test for continuous variables 
Fig. 1 Incidence of AML 2012 2014 in the German AMLSG BiO registry $(N=3251)$ compared to selected German cancer registries and the US SEER program. AML in the cancer registries Bavaria, Saarland, and North RhineWestphalia (NRW) and US Surveillance, Epidemiology, and End Results (SEER) 2009-2013
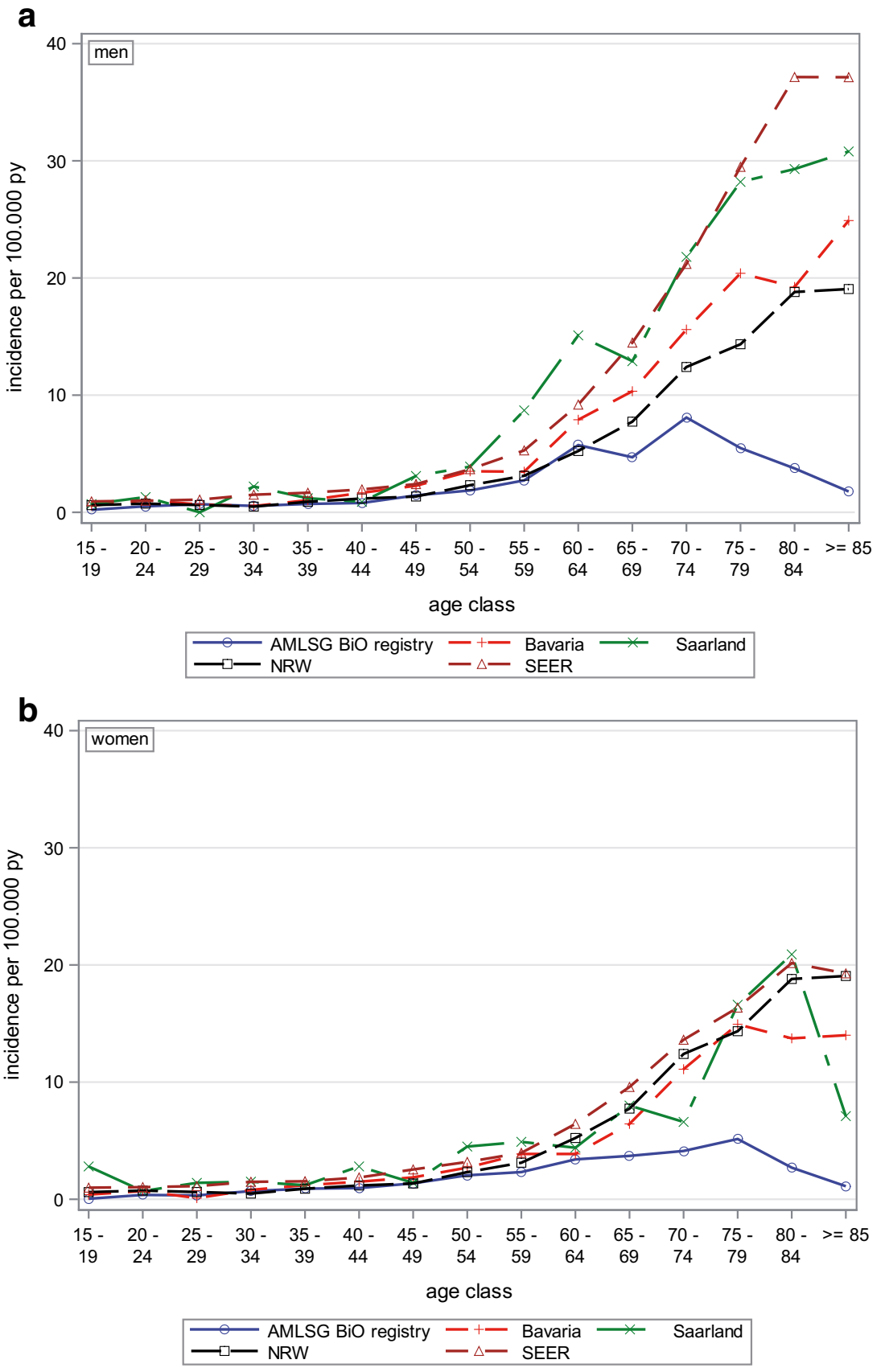

comorbidity index, ECOG performance status, and activating FLT3 mutations are shown in Fig. 3 a-d. The figures show absolute numbers on the Y-axis and percentages in relation to the total number per age group in the bars. Overall, most patients were in the age group $\geq 70$ years. The distribution of ELN risk groups changed significantly ( $p$ value $<0.001$ ) with increasing age. In younger patients, the favorable risk group was either the most frequent (age $<45$ years) or the second most frequent group (age 45-59 years), whereas in older patients (age 60-69 years, age $>70$ years) the favorable risk group was the smallest subgroup. However, the absolute number of patients in the favorable risk group ranged between
65 and 192 patients and was comparable in all age groups (Fig. 3a). Thus, the relative prevalence of favorable-risk AML decreased whereas the absolute prevalence remained stable with increasing age. In contrast, the absolute and relative numbers of patients in the adverse risk group consistently increased with increasing age. Overall, most patients (39\%) presented with no comorbidity burden (HCT-CI 0 ) with nearly stable absolute numbers across the different age groups, whereas the absolute and relative prevalence of patients with limited (HCT-CI 1-2) and extensive (HCT-CI $\geq 3$ ) HCT comorbidity index increased with increasing age (Fig. 3b). A similar relationship was present for the ECOG performance 
Fig. 2 Distribution of AML subtypes $(N, \%)$ according to the WHO 2008 classification in 2740 patients. Abbreviations: CEBPA CCAAT/enhancer-binding protein alpha, $N$ number of patients, NPM1 nucleophosmin-1

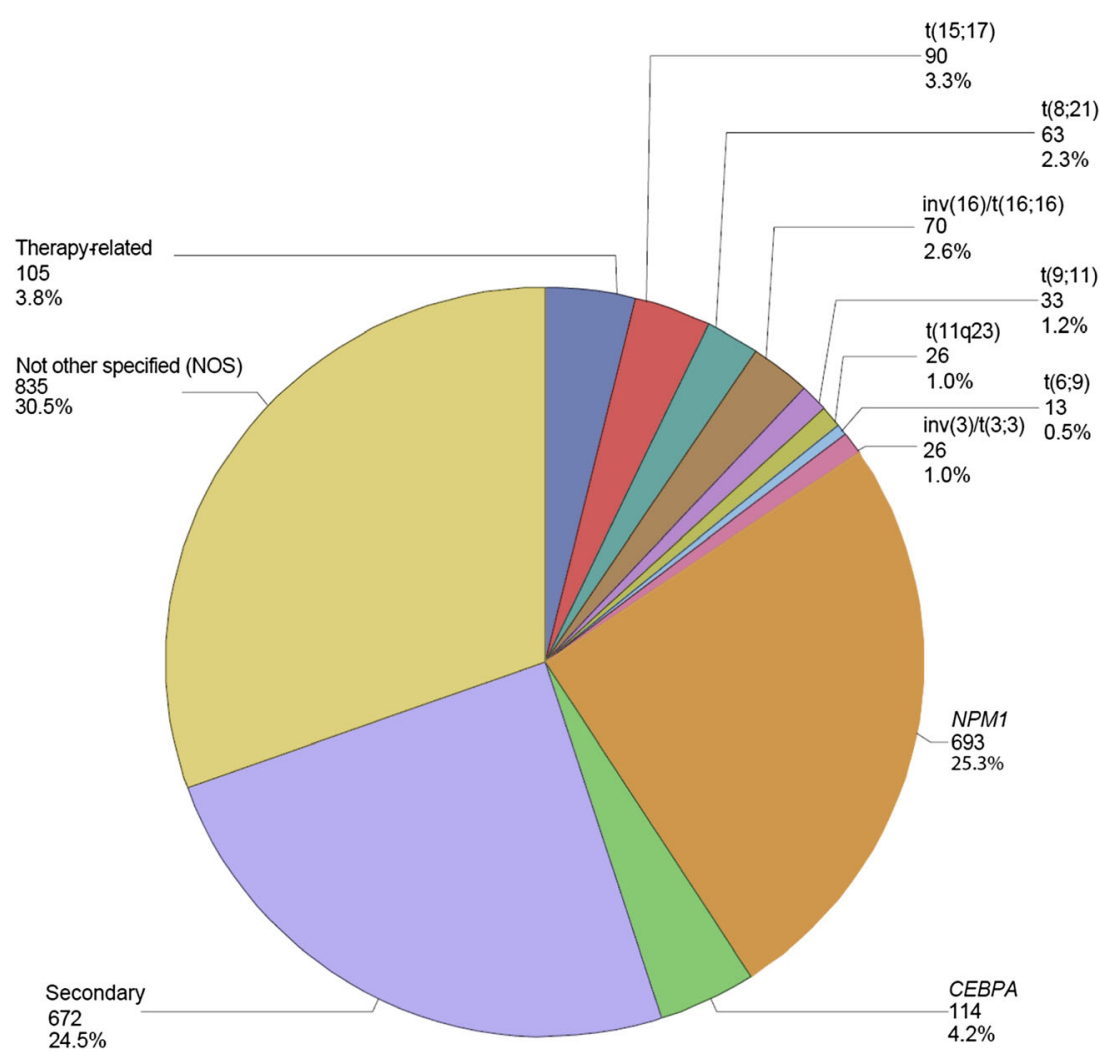

status with a nearly stable absolute prevalence but decreasing relative frequency of fully active (ECOG 0) patients, whereas patients with slightly to moderately diminished performance status (ECOG 1/2) represented the largest group in older age groups (age 60-69 years, age $\geq 70$ years) (Fig. 3c). Based on the performance status $(\mathrm{ECOG} \leq 2)$, most of our patients were eligible for intensive chemotherapy even in the age group $\geq 70$ years.

We were also interested in the distribution of patients with activating FLT3-ITD and FLT3-TKD mutations due to potentially available targeted therapy [14]. Although the relative numbers decreased significantly with increasing age, the absolute number of patients with activating FLT3 mutations increased with age ( $<45$ years, $n=135 ; 45-59$ years, $n=245$; 60-69 years, $n=203$; $\geq 70$ years, $n=271$; Fig. $3 d$ ). Based on reported very good response rates achieved in NPM1-mutated AML with intensive induction therapy [9-12], we performed an additional analysis according to NPM1 mutational status and age. The percentages (absolute numbers) of patients with NPM1 mutations according to the four age groups in ascending order were $24 \%(n=100), 34 \%(n=281), 27 \%$ ( $n=238)$, and $21 \%(n=295)$ indicating that even in age group $\geq 70$ years a substantial number of patients could potentially benefit from intensive induction chemotherapy (suppl. Figure 3).
Most of the patients with information on treatment $(N=3213)$ received intensive chemotherapy $(71.1 \%$, Table 1). However, starting with the age of 65 years, a substantial and increasing proportion of patients received either non-intensive therapy or best supportive care (BSC) (Fig. 4) reaching a proportion of more than $50 \%$ in patients $>75$ years.

In multivariate models (Table 2 ), age $>70$ years was the strongest predictor to receive non-intensive treatment (OR, 9.91; 95\% CI, 7.08-13.86; reference, intensive chemotherapy) or BSC (OR, 4.78; 95\% CI, 3.17-7.22; reference, intensive chemotherapy), whereas younger age $(<60$ years) was inversely associated with intensive chemotherapy. Other predictors for non-intensive treatment or BSC were ELN adverse-risk disease, comorbidity with an HCT-CI index $\geq 3$, and impaired performance status (ECOG 2-4). With increasing comorbidity index, the association with nonintensive treatment (HCT-CI 1-2 vs. 0: OR, 1.30; 95\% CI, 0.96-1.77; HCT-CI $\geq 3$ vs. 0: OR, 1.65; 95\% CI, 1.20-2.65) and BSC (HCT-CI 1-2 vs. 0: OR, 1.66; 95\% CI, 1.10-2.57; HCT-CI $\geq 3$ vs. 0: OR, $2.61 ; 95 \%$ CI, 1.68-4.07, respectively) became stronger. Of note, after adjustment for the other covariates, ELN favorable risk was only rarely associated with BSC as treatment 

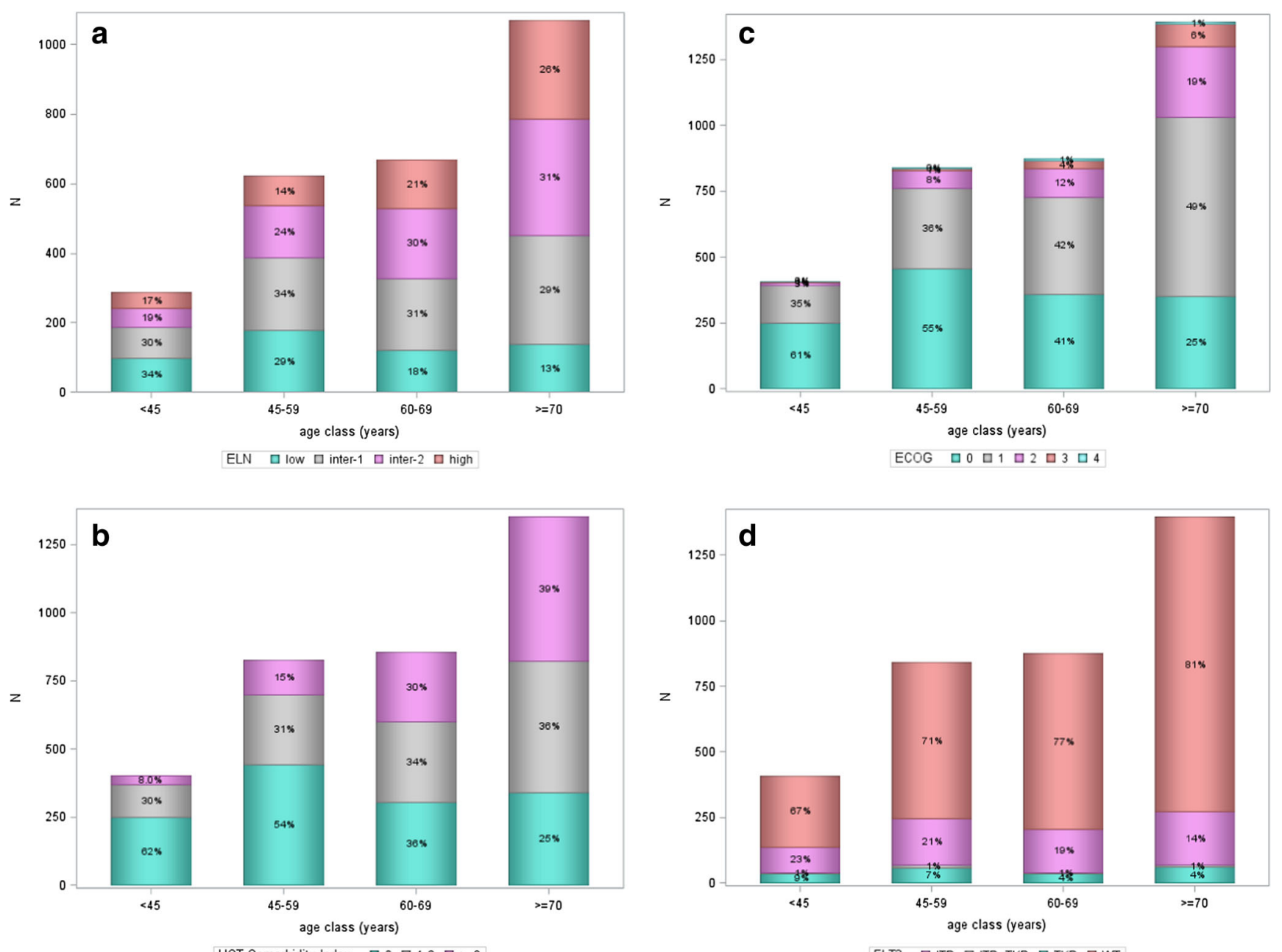

Fig. 3 a-d 2010 European LeukemiaNet (ELN) classification, HCT comorbidity index, performance status (ECOG), and FLT3 mutations by age classes in the AMLSG $\mathrm{BiO}$ registry $(N=3521)$. Abbreviations: ELN European LeukemiaNet, ECOG Eastern Cooperative Oncology

Group performance status, FLT3 FMS-related tyrosine kinase 3, HCT hematopoietic cell transplantation, ITD internal tandem duplication, $N$ number of patients, NPM1 nucleophosmin-1, TKD tyrosine kinase domain, $W T$ wild type

Fig. 4 Frequency of treatment strategy (intensive, non-intensive and best supportive care (BSC)) according to age. Abbreviation: BSC, best supportive care ; $\mathrm{N}$, number of patients

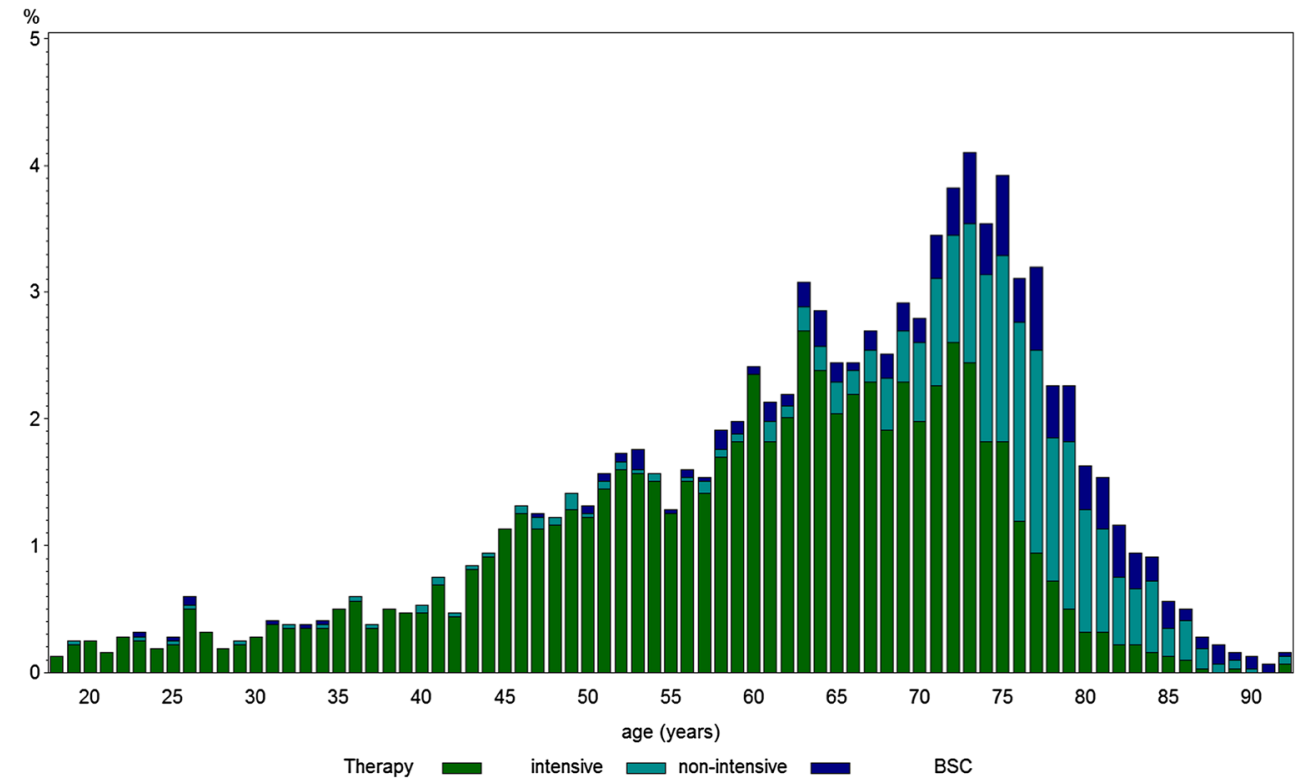


Table 2 Multinomial logistic regression for therapy, sex, age classes, 2010 ELN classification, ECOG performance status, HCT comorbidity index, and BMI

\begin{tabular}{|c|c|c|c|}
\hline \multirow[t]{3}{*}{ Covariate } & & \multicolumn{2}{|l|}{$\begin{array}{l}\text { Intensive therapy (reference) } \\
(N=1654)\end{array}$} \\
\hline & & Non-intensive $^{\mathrm{a}}(N=460)$ & $\operatorname{BSC}(N=207)$ \\
\hline & & Odds ratio $(95 \% \mathrm{CI})$ & Odds ratio $(95 \% \mathrm{CI})$ \\
\hline \multirow[t]{3}{*}{ Age class (years) } & $<60$ & $0.51(0.31,0.84)$ & $0.76(0.43,1.34)$ \\
\hline & $60-69$ & 1 & 1 \\
\hline & $\geq 70$ & $9.91(7.08,13.86)$ & $4.78(3.17,7.22)$ \\
\hline \multirow[t]{2}{*}{ Sex } & Woman & $0.97(0.76,1.24)$ & $0.89(0.64,1.23)$ \\
\hline & Men & 1 & 1 \\
\hline \multirow[t]{4}{*}{ ELN 2010 classification } & Favorable & $0.95(0.65,1.39)$ & $0.39(0.21,0.71)$ \\
\hline & Intermediate-1 & 1 & 1 \\
\hline & Intermediate- 2 & $1.10(0.81,1.51)$ & $1.13(0.76,1.67)$ \\
\hline & Adverse & $1.60(1.16,2.23)$ & $1.17(0.76,1.79)$ \\
\hline \multirow[t]{3}{*}{ HCT comorbidity index } & 0 & 1 & 1 \\
\hline & $1-2$ & $1.30(0.96,1.77)$ & $1.66(1.10,2.57)$ \\
\hline & $\geq 3$ & $1.65(1.20,2.26)$ & $2.61(1.68,4.07)$ \\
\hline \multirow[t]{2}{*}{ ECOG } & $0-1$ & 1 & 1 \\
\hline & $2-4$ & $1.94(1.42,2.65)$ & $4.31(3.01,6.16)$ \\
\hline \multirow[t]{3}{*}{ BMI $\left(\mathrm{kg} / \mathrm{m}^{2}\right)$} & $<20$ & $1.58(0.91,2.76)$ & $1.46(0.77,2.78)$ \\
\hline & $20-24$ & 1 & 1 \\
\hline & $\geq 25$ & $0.86(0.67,1.12)$ & $0.57(0.40,0.80)$ \\
\hline
\end{tabular}

$B M I$ body mass index, $B S C$ best supportive care, $C I$ confidence interval, ECOG Eastern Cooperative Oncology Group performance status, ELN European LeukemiaNet, $H C T$ hematopoietic cell transplantation, $N$ number of patients

${ }^{\text {a }}$ Including azacitidine, decitabine, and low-dose cytarabine strategy (OR 0.39 95\% CI, 0.21-0.71), whereas nonintensive chemotherapy was nearly as frequently chosen as intensive chemotherapy (OR $0.9595 \% \mathrm{CI}, 0.65-1.39$ ). Compared to BMI $20-24 \mathrm{~kg} / \mathrm{m}^{2}$, obesity was less frequently associated with non-intensive treatment (OR, 0.86 ; 95\% CI, 0.67-1.12) and BSC (OR, 0.57; 95\% CI, $0.40-0.80)$.

\section{Discussion}

Based on the absolute numbers of 1307 patients included in 2014, the academic population-based AMLSG BiO registry study roughly represents one third of expected cases per year with newly diagnosed AML in Germany and Austria with an assumed incidence rate of 4 of 100,000 inhabitants. However, compared to other population-based cancer registries, the agespecific incidence rate of the AMLSG BiO registry is lower for persons of older age. Compared to the data of other cancer registries, about $20 \%$ of men aged over 70 years and women aged over 75 years with AML were registered in the AMLSG $\mathrm{BiO}$ registry suggesting that older AML patients were underreported. Possible explanations are that older patients were less frequently referred to specialized leukemia-treating hospitals and that in older AML patients genetic information was considered less relevant for the treatment decision. However, median age of 65 years at diagnosis in our AMLSG BiO registry was consistent with data reported from the Netherlands with a comparable population [17]. Thus, data from our AMLSG BiO registry shows an excellent and good population representation up to the age of 70 years and with an age between 70 and 80 years, respectively.

In our population-based approach, the distribution of selected genetic markers such as activating FLT3 mutations and mutated NPM1 differed in a clinically relevant manner from that recently reported in a large group of patients, all of them treated in interventional clinical trials with a median age below 60 years [8]; interestingly, the occurrence of NPM1 mutations was very similar whereas the number of activating FLT3 mutations was lower by a factor of 1.32 . This clearly reflects patient selection towards younger and fitter patients when treated in clinical trials. As illustrated in Fig. 3d with a nearly stable absolute prevalence of patients exhibiting activating FLT3 mutations in all age groups, the relative prevalence significantly decreased from $33,29,24$, to 
$19 \%$ in the age groups $<45,45-59,60-69$, and $\geq 70$ years. This observation is consistent with findings showing that the frequency of FLT3-ITD and FLT3-TKD in adults decreases with increasing age [29]. However, with regard to the public health perspective and the planning of future clinical trials with FLT3 inhibitors, our results provide a good data basis in terms of expected absolute and relative numbers in the different age groups showing that the absolute number of older patients with activating FLT3 mutations ( $\geq 60$ years) still exceeds that observed in younger patients $(<60$ years).

In the AMLSG $\mathrm{BiO}$ registry, most patients with AML are older with a high proportion of patients with adverse genetics (Fig. 3a), presence of comorbidities (Fig. 3b), and impaired performance status (Fig. 3c). However, up to the age of 75 years, most patients receive intensive chemotherapy (Fig. 4), while beyond this age more patients are treated with hypomethylating agents, low-dose cytarabine, or best supportive care. Consistent with the literature, in our study, impaired performance status and increased comorbidity index were more frequent in older age [30]. There has been a discussion about the cofactors influencing the treatment decision such as performance status and the burden of comorbidities [3] as well as the genetic profile of the disease [9]. Due to all these factors, the treatment of AML in older patients remains quite challenging [30], since intensive chemotherapy is toxic and less well tolerated by the older AML patients. Recent developments of targeted treatment for AML patients with activating FLT3 mutation have drawn the attention to this subgroup, which comprises about one quarter of our registry patients. Although the relative number of activating FLT3 mutation decreases with increasing age, still a substantial proportion of patients above the age of 60 years may benefit from specific FLT3 inhibitors [31]. Thus, the differences of the genetic and clinical profiles between older and younger AML patients have to be taken into account when treatment strategies are developed [32]. A substantial proportion of older AML patients exhibit a NPM1 mutation, which predicts for high response rates to intensive induction therapy even in the older patients with complete remission (CR) rates as high as $80 \%$ [33, 34]. This genetic marker information may therefore be used to guide treatment strategy in older patients.

After adjusting for disease and performance (ECOG, HCTCI) parameters, we found overweight to be associated with the intensive treatment approach rather than non-intensive treatment and BSC. This suggests that obese patients are considered to tolerate intensive chemotherapy better compared to normal or underweight patients. This observation is paralleled by the finding that obese older AML patients have a better survival [35]. Whether the higher treatment intensity or other factors are responsible for superior survival of obese older patients remains elusive.
In conclusion, our study characterizes the academic population-based AMLSG BiO registry, which has excellent and good population coverage up to the age of 70 years and between 70 and 80 years, respectively. Our study indicates that the distribution of the genetic profile differs in a clinically fashion relevant by age. Taking into account relative and absolute numbers by age group, our study provides valid data for public health evaluations and the planning of interventional studies in genetically defined subgroups.

Acknowledgements We thank the cancer registries in Bavaria, North Rhine-Westphalia, and Saarland for the selection of the state-specific AML cases.

Austria: David Nachbaur M.D., Günter Gastl M.D., Universitätsklinikum Innsbruck, Innsbruck; Andreas Petzer M.D., Krankenhaus der Barmherzigen Schwestern Linz, Linz; Elisabeth Koller M.D., Hanuschkrankenhaus, Wien.

Germany: Günter Schlimok M.D., Klinikum Augsburg, Augsburg; Jörg Westermann M.D., Charité Campus Virchow-Klinikum, Berlin; Peter Brossart M.D., Marie von Lilienfeld-Toal M.D., Universitätsklinikum Bonn, Bonn; Jürgen Krauter M.D., Jens Kersten M.D., Städtisches Klinikum, Braunschweig; Rainer Haas M.D., Andrea Kündgen M.D. Universitätsklinikum Düsseldorf, Düsseldorf; Peter Reimer M.D., Mohammed Wattad M.D., Kliniken Essen Süd, Ev. Krankenhaus EssenWerden gGmbH, Essen; Michael Lübbert M.D., Universitätsklinikum Freiburg, Freiburg; Alexander Burchardt M.D., Matthias Rummel M.D., Universitätsklinikum Gießen, Gießen; Gerald Wulf M.D., Lorenz Trümper M.D., Universitätsklinikum Göttingen, Göttingen; Hans Salwender M.D., Asklepios Klinik Altona, Hamburg; Arnold Ganser M.D., Michael Heuser M.D. Brigitte Schlegelberger M.D., Medizinische Hochschule Hannover, Hannover; Michael Pfreundschuh M.D., Gerhard Held M.D., Universitätsklinikum des Saarlandes, Homburg; Mark Ringhoffer M.D., Martin Bentz M.D., Städtisches Klinikum Karlsruhe gGmbH, Karlsruhe; Heinz A. Horst M.D., Michael Kneba M.D., Universitätsklinikum Schleswig-Holstein, Campus Kiel, Kiel; Stephan Kremers M.D., CaritasKrankenhaus Lebach, Lebach; Gerhard Heil M.D., Klinikum Lüdenscheid, Lüdenscheid; Thomas Fischer M.D. / Thomas Heinicke M.D. Universitätsklinikum Magdeburg A. ö. R, Magdeburg, Germany; Thomas Kindler M.D., Matthias Theobald M.D., Universitätsklinikum Mainz, Mainz; Katharina Götze M.D., Christian Peschel M.D., Klinikum rechts der Isar der Technischen Universität München, München; Sabine Struve M.D., Clemens Wendtner M.D., Klinikum Schwabing, München; ClausHenning Köhne M.D., Klinikum Oldenburg, Oldenburg; Axel Matzdorff M.D., Caritas-Klinik St. Theresia, Saarbrücken; Hans-Günther Mergenthaler M.D., Klinikum Stuttgart, Stuttgart; Heinz Kirchen M.D., Krankenhaus der Barmherzigen Brüder, Trier; Helmut R. Salih M.D., Lothar Kanz M.D., Universitätsklinikum Tübingen, Tübingen; Hartmut Döhner M.D., Konstanze Döhner M.D., Richard F. Schlenk M.D., Universitätsklinikum Ulm, Ulm; Aruna Raghavachar M.D., HeliosKlinikum Wuppertal, Wuppertal.

Author's contribution Conception and design: GN and RFS. Provision of study materials or patients: all authors. Collection and assembly of data: GN, EF, SE, DW, and RFS. Data analysis and interpretation: GN, EF, SE, HD, and RFS. Manuscript writing: GN, HD, and RFS. Final approval of manuscript: all authors.

\section{Compliance with ethical standards}

The study was approved by the ethical review boards of all participating centers. 
Conflict of interest The authors declare that they have no conflict of interest.

Open Access This article is distributed under the terms of the Creative Commons Attribution 4.0 International License (http:// creativecommons.org/licenses/by/4.0/), which permits unrestricted use, distribution, and reproduction in any medium, provided you give appropriate credit to the original author(s) and the source, provide a link to the Creative Commons license, and indicate if changes were made.

\section{References}

1. SEER Cancer Statistics Review, 1975-2009 - Previous versionSEER Cancer Statistics Review. https://seer.cancer.gov/archive/csr/ 19752009 pops09/. Accessed 5 Dec 2016

2. Cancer Statistics Review, 1975-2013-SEER Statistics. https:// seer.cancer.gov/csr/1975 2013/. Accessed 5 Dec 2016

3. Klepin HD, Rao AV, Pardee TS (2014) Acute myeloid leukemia and myelodysplastic syndromes in older adults. J Clin Oncol 32: 2541-2552. https://doi.org/10.1200/JCO.2014.55.1564

4. Juliusson G, Lazarevic V, Hörstedt A-S et al (2012) Acute myeloid leukemia in the real world: why population-based registries are needed. Blood 119:3890-3899. https://doi.org/10.1182/blood2011-12-379008

5. Ocias LF, Larsen TS, Vestergaard H et al (2016) Trends in hematological cancer in the elderly in Denmark, 1980-2012. Acta Oncol 55(Suppl 1):98-107. https://doi.org/10.3109/0284186X.2015. 1115124

6. L Bullinger, Döhner K, Döhner H (2017) Genomics of acute myeloid leukemia diagnosis and pathways. J Clin Oncol. in press

7. Nagel G, Stocks T, Späth D et al (2012) Metabolic factors and blood cancers among 578,000 adults in the metabolic syndrome and cancer project (Me-Can). Ann Hematol 91:1519-1531. https://doi.org/ 10.1007/s00277-012-1489-z

8. Papaemmanuil E, Gerstung M, Bullinger L et al (2016) Genomic classification and prognosis in acute myeloid leukemia. N Engl $\mathrm{J}$ Med 374:2209-2221. https://doi.org/10.1056/NEJMoa1516192

9. Schlenk RF, Döhner H (2013) Genomic applications in the clinic: use in treatment paradigm of acute myeloid leukemia. Hematology Am Soc Hematol Educ Program 2013:324-330. https://doi.org/10. 1182/asheducation-2013.1.324

10. Döhner H, Weisdorf DJ, Bloomfield CD (2015) Acute myeloid leukemia. N Engl J Med 373:1136-1152. https://doi.org/10.1056/ NEJMra1406184

11. Döhner H, Estey EH, Amadori S et al (2010) Diagnosis and management of acute myeloid leukemia in adults: recommendations from an international expert panel, on behalf of the European LeukemiaNet. Blood 115:453-474. https://doi.org/10.1182/blood2009-07-235358

12. Schlenk RF, Döhner K, Krauter J et al (2008) Mutations and treatment outcome in cytogenetically normal acute myeloid leukemia. $\mathrm{N}$ Eng1 J Med 358:1909-1918. https://doi.org/10.1056/ NEJMoa074306

13. Döhner H, Estey E, Grimwade D et al (2017) Diagnosis and management of AML in adults: 2017 ELN recommendations from an international expert panel. Blood 129:424-447. https://doi.org/10. 1182/blood-2016-08-733196

14. Stone RM, Mandrekar S, Sanford BL et al (2015) The multi-kinase inhibitor midostaurin (M) prolongs survival compared with placebo $(\mathrm{P})$ in combination with daunorubicin (D)/cytarabine $(\mathrm{C})$ induction (ind), high-dose $\mathrm{C}$ consolidation (consol), and as maintenance (maint) therapy in newly diagnosed acute myeloid leukemia (AML) patients (pts) age 18-60 with FLT3 mutations (muts): an international prospective randomized (rand) $\mathrm{P}$ controlled double-blind trial (CALGB 10603/RATIFY [Alliance]). Blood 126:6

15. Gerstung M, Papaemmanuil E, Martincorena I et al (2017) Precision oncology for acute myeloid leukemia using a knowledge bank approach. Nat Genet. https://doi.org/10.1038/ng.3756

16. Ostgård LSG, Nørgaard JM, Severinsen MT et al (2013) Data quality in the Danish National Acute Leukemia Registry: a hematological data resource. Clin Epidemiol 5:335-344. https://doi.org/10. 2147/CLEP.S48411

17. Dinmohamed AG, Visser O, van Norden Y et al (2016) Treatment, trial participation and survival in adult acute myeloid leukemia: a population-based study in the Netherlands, 1989-2012. Leukemia 30:24-31. https://doi.org/10.1038/leu.2015.188

18. Sant M, Minicozzi P, Mounier M et al (2014) Survival for haematological malignancies in Europe between 1997 and 2008 by region and age: results of EUROCARE-5, a population-based study. Lancet Oncol 15:931-942. https://doi.org/10.1016/S14702045(14)70282-7

19. Pulte D, Jansen L, Castro FA, Brenner H (2016) Changes in the survival of older patients with hematologic malignancies in the early 21 st century. Cancer 122:2031-2040. https://doi.org/10. $1002 /$ cncr.30003

20. Burnett A, Wetzler M, Löwenberg B (2011) Therapeutic advances in acute myeloid leukemia. J Clin Oncol 29:487-494. https://doi. org/10.1200/JCO.2010.30.1820

21. Oken MM, Creech RH, Tormey DC et al (1982) Toxicity and response criteria of the Eastern Cooperative Oncology Group. Am J Clin Oncol 5:649-655

22. Sorror ML, Maris MB, Storb R et al (2005) Hematopoietic cell transplantation (HCT)-specific comorbidity index: a new tool for risk assessment before allogeneic HCT. Blood 106:2912-2919. https://doi.org/10.1182/blood-2005-05-2004

23. Vardiman JW, Thiele J, Arber DA et al (2009) The 2008 revision of the World Health Organization (WHO) classification of myeloid neoplasms and acute leukemia: rationale and important changes. Blood 114:937-951. https://doi.org/10.1182/blood-2009-03209262

24. Mitelman F ISCN 1995 An international system for human cytogenetic nomenclature (1995), 1995. Karger, Basel

25. Schlenk RF, Taskesen E, van Norden Y et al (2013) The value of allogeneic and autologous hematopoietic stem cell transplantation in prognostically favorable acute myeloid leukemia with double mutant CEBPA. Blood 122:1576-1582. https://doi.org/10.1182/ blood-2013-05-503847

26. Taskesen E, Bullinger L, Corbacioglu A et al (2011) Prognostic impact, concurrent genetic mutations, and gene expression features of AML with CEBPA mutations in a cohort of 1182 cytogenetically normal AML patients: further evidence for CEBPA double mutant AML as a distinctive disease entity. Blood 117:2469-2475. https:// doi.org/10.1182/blood-2010-09-307280

27. Statistisches Bundesamt (2015) Bevölkerung 2012-2014. Statistisches Bundesamt Destatis, https://www-genesis.destatis. de/genesis/online/link/tabellen/12411*

28. Surveillance, Epidemiology, and End Results (SEER) (2015) AML incidence 2009-2013. National cancer Institute, http://seer. cancer.gov/faststats/selections.php?\#Output

29. Schneider F, Hoster E, Schneider S et al (2012) Age-dependent frequencies of NPM1 mutations and FLT3-ITD in patients with normal karyotype AML (NK-AML). Ann Hematol 91:9-18. https://doi.org/10.1007/s00277-011-1280-6

30. Sanz MA, Iacoboni G, Montesinos P, Venditti A (2016) Emerging strategies for the treatment of older patients with acute myeloid leukemia. Ann Hematol 95:1583-1593. https://doi.org/10.1007/ s00277-016-2666-2 
31. Schlenk RF, Fiedler W, Salih HR et al (2016) Impact of age and midostaurin-dose on response and outcome in acute myeloid leukemia with FLT3-ITD: interim-analyses of the AMLSG 16-10 Trial. Blood 128:449

32. Tsai C-H, Hou H-A, Tang J-L et al (2016) Genetic alterations and their clinical implications in older patients with acute myeloid leukemia. Leukemia 30:1485-1492. https://doi.org/10.1038/leu.2016.65

33. Schlenk RF, Döhner K, Kneba M et al (2009) Gene mutations and response to treatment with all-trans retinoic acid in elderly patients with acute myeloid leukemia. Results from the AMLSG Trial AML HD98B. Haematologica 94:54-60. https://doi.org/10.3324/ haematol.13378
34. Becker H, Marcucci G, Maharry K et al (2010) Favorable prognostic impact of NPM1 mutations in older patients with cytogenetically normal de novo acute myeloid leukemia and associated gene- and microRNA-expression signatures: a Cancer and Leukemia Group B study. J Clin Oncol 28:596-604. https://doi.org/10.1200/JCO.2009. 25.1496

35. Brunner AM, Sadrzadeh H, Feng Y et al (2013) Association between baseline body mass index and overall survival among patients over age 60 with acute myeloid leukemia. Am J Hematol 88:642-646. https://doi.org/10.1002/ajh.23462 